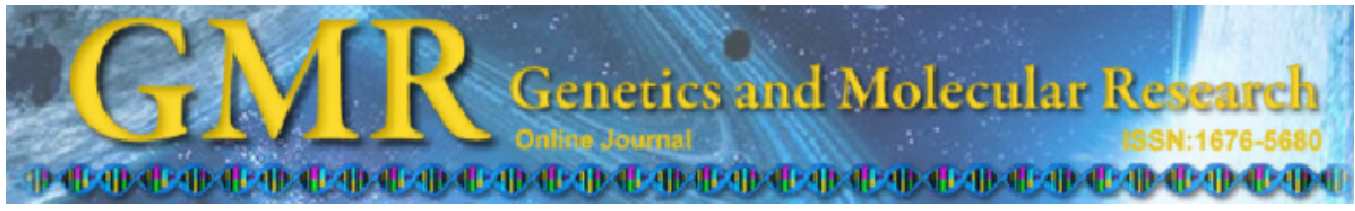

\title{
A new single-nucleotide mutation (rs362719) of the reelin $(R E L N)$ gene associated with schizophrenia in female Chinese Han
}

\author{
W.J. Kuang ${ }^{1,2}$, R.F. Sun ${ }^{1,2}$, Y.S. Zhu ${ }^{1,3}$ and S.B. Li ${ }^{1,2}$ \\ ${ }^{1}$ Department of Forensic Sciences, College of Medicine, \\ Xi'an Jiaotong University, Xi'an, Shaanxi, P.R. China \\ ${ }^{2}$ Key Laboratory of Environment and Gene Related Diseases, \\ Xi'an Jiaotong University, Ministry of Education, Xi'an, Shaanxi, P.R. China \\ ${ }^{3}$ Key Laboratory of Reproduction and Genetics of Ningxia Hui Autonomous Region, \\ Yinchuan, P.R. China
}

Correspondence author: S.B. Li

E-mail: shbinlee@mail.xjtu.edu.cn

Genet. Mol. Res. 10 (3): 1650-1658 (2011)

Received February 22, 2011

Accepted May 17, 2011

Published August 5, 2011

DOI http://dx.doi.org/10.4238/vol10-3gmr1343

\begin{abstract}
Reelin is an extracellular signaling protein that plays an important role in the development of the central nervous system. Postmortem studies have shown lower reelin protein levels in the brains of patients with schizophrenia and bipolar disorder compared with controls. Genetic studies have also shown that mutations in the reelin gene $(R E L N)$ increase the risk for schizophrenia and bipolar disorder. We evaluated whether an RELN gene variant, rs362719, which has been associated with increased susceptibility to bipolar disorder, is also associated with susceptibility to schizophrenia. We included 405 Chinese Han schizophrenia patients and 390 controls in our study. The polymorphism was genotyped by PCR and RFLP methods. We found a significant difference in allele frequency distribution $(\mathrm{P}<0.05)$ between schizophrenia patients and controls. The frequency of the A allele was significantly higher in schizophrenia patients than in healthy controls.
\end{abstract}


The effect of SNP rs362719 on allele distribution was significant in female $(\mathrm{P}<0.05)$ but not in male participants $(\mathrm{P}=0.473)$. Besides the gender factor, demographic and clinical characteristics of the rs362719 genotype groups were also analyzed using the chi-square test, but no significant differences were found. We conclude that rs362719 of the $R E L N$ gene is associated with susceptibility to schizophrenia in Chinese Han, possibly through a gender-specific mechanism. Further studies will be needed to confirm this genetic risk factor for schizophrenia.

Key words: Schizophrenia; Bipolar disorder; RELN; Polymorphism

\section{INTRODUCTION}

Reelin (RELN) is an extracellular matrix protein coded by a gene located on chromosome $7 \mathrm{q} 22$. RELN is expressed in the brain, notably not only in the cerebral cortex and the spinal cord, but also in other organs in the body. RELN plays a pivotal role in the regulation of neuronal migration, cortical lamination and positioning in the developing brain. Reelin is expressed primarily in the brain and the spinal cord and is also found in other organs and tissues in the body, notably in the cerebral cortex. It has dual roles in mammalian brain. During embryonic brain development, reelin is secreted by Cajal-Retzius cells of the marginal zone and provides a signal for proper migration of new post-mitotic neurons to form distinct brain areas (Quattrocchi et al., 2002). In the adult brain, reelin is very rich in GABAergic interneurons of the hippocampus, where it has been found to play an important role in neuroplasticity and long-term potentiation by modulating NMDA receptor-mediated neurotransmission, which is necessary for synaptic plasticity, learning and memory (Chen et al., 2005). Hence, an altered reelin level may cause deficits in neuronal development and function. Indeed, reelin binds directly to one of two receptors, a very low-density lipoprotein receptor (VLDLR) and ApoE receptor type 2 (ApoER2), setting off a phosphorylation cascade of Dab1. This signaling pathway controls cell positioning in the developing brain and adult neurogenesis (Herz and Chen, 2006). Thus, abnormal reelin might play a pathogenic role in neuropsychiatric illnesses.

Reelin is implicated in pathogenesis of several brain disorders such as schizophrenia and bipolar disorder. One study indicated that the 7q22 region has been implicated in schizophrenia and bipolar disorder by a joint meta-analysis method (Badner and Gershon, 2002b). A substantial amount of evidence has shown that reelin mRNA expression and protein levels are decreased in GABAergic neurons in the brain, such as prefrontal cortex, cerebellar, hippocampus, and basal ganglia in schizophrenia and bipolar disorder patients (Fatemi et al., 2000; Guidotti et al., 2000; Veldic et al., 2007). These results suggest that reelin expression may be associated with a susceptibility to both disorders. The hypothesis is that the reduced reelin mRNA expression was due to hypermethylation of the $\mathrm{CpG}$ island, which is located upstream from the RELN promoter sequence. Hypermethylation was observed in the postmortem prefrontal cortex of schizophrenia cases compared with controls (Abdolmaleky et al., 2005; Grayson et al., 2005). In addition, some pharmacological agents used in the clinical treatment of schizophrenia (Dong et al., 2008) and bipolar disorder (Dong et al., 2007) have been shown to reverse this hypermethylation of the $\mathrm{CpG}$ island upstream from the RELN promoter. However, this observation was not confirmed by some other studies (Mill et al., 
2008; Tochigi et al., 2008). These contradictory findings in schizophrenia patients suggest that hypermethylation of the RELN promoter was not the only cause that contributed to the development of schizophrenia.

To our knowledge, schizophrenia and bipolar disorder have been considered to be nosologically and etiologically distinct disorders in DSM-IV and ICD-10 classification, which was based on the Kraepelinian dichotomy. However, diagnosis of these disorders is based primarily on symptoms that overlap with each other and the above hypothesis has been challenged by recent progress in many psychiatric biological studies (van Os et al., 1999; Moller, 2003; Murray et al., 2004; Craddock and Owen, 2005). Genome-wide linkage screenings have shown some overlap between schizophrenia and bipolar disorder (Berrettini, 2003; Tsuang et al., 2004; Moskvina et al., 2009). Many family and twin studies as well as molecular-genetic studies have proposed some etiological overlap, which was at least partially due to genetic factors (Lander and Kruglyak, 1995; Cardno et al., 2002). Meta-analyses were performed for schizophrenia as well as for bipolar disorder. The results showed the overlap of some candidate regions in the chromosomes, such as 13q and 22q (Badner and Gershon, 2002a; Berrettini, 2003). Schizophrenia and bipolar disorder have also demonstrated some similarities in neurotransmitter dysfunction (Clinton and Meador-Woodruff, 2004).

Heritability has been estimated to be responsible for at least $80 \%$ of schizophrenia and bipolar disorders (Cardno and Gottesman, 2000; McGuffin et al., 2003). Therefore, genetic research is a main topic in the study of both disorders. Direct evidence from a Swedish population study showed a high degree of genetic overlap between these two disorders, according to a series of 76,472 people with bipolar disorder and/or schizophrenia taken from a Swedish medical registry. It was found that the two disorders share $63.4 \%$ of their genetic determinants (Lichtenstein et al., 2009). Some of the loci implicated in bipolar disorder may also be detectable in schizophrenia patients, and vice versa. Based on the above hypothesis that the overlap genes share a common background cause in schizophrenia and bipolar disorder, it is important to explore the genetic associations of the susceptibility locus in both bipolar disorder and schizophrenia patients.

In a genome-wide association study (GWAS), a single nucleotide polymorphism (SNP) in RELN gene (rs7341475) was found to increase the risk of schizophrenia in women, but not in men (Shifman et al., 2008). Allelic variations of RELN have also been found to be correlated with working memory, memory and executive functioning in nuclear families where one of the members suffered from schizophrenia (Wedenoja et al., 2008). Despite the efforts to identify the cause of schizophrenia, it remains elusive as regards the definitive causes of the disorder. Another research has identified another variant (rs362719) of RELN gene, which has been found to increase the susceptibility to bipolar disorder patients in women (Goes et al., 2010). Based on the hypothesis that the overlap gene shares a similar background in both schizophrenia and bipolar disorder, we conducted a case-control study of this SNP locus (rs362719) to test the possible association with the schizophrenia in various Chinese populations.

\section{MATERIAL AND METHODS}

\section{Subjects}

All ethnic Han Chinese subjects were recruited from Shaanxi, a Midwest province in China. A total of 405 unrelated patients were enrolled in this study (205 males: mean age \pm SD, 
$42.9 \pm 11.7$ years; 200 females: $42.1 \pm 12.6$ years). To confirm the disease type, each patient was diagnosed by at least two independent psychiatrists. The diagnosis was carried out strictly on the basis of the criteria for schizophrenia outlined by Diagnostic and Statistical Manual of Mental Disorders, 4th edition (American Psychiatric Association, 2000). All patients had no previous history of substance abuse or organic mental disorders. A final diagnosis was made by two another experienced psychiatrists from the Department of Psychiatry, Xi'an Jiaotong University on the basis of the interview reports and medical records. The control group consisted of 390 unrelated healthy volunteers (194 males: mean age \pm SD, $41.6 \pm 14.0$ years; 196 females: $42.0 \pm 13.4$ years). None of the controls had a family history (first-degree relatives) of psychiatric illness or substance abuse. All control subjects were interviewed by one psychiatrist and were psychiatrically screened on the basis of unstructured interviews. Informed consent was obtained from all participants for the genetic study. This study was approved by the Ethics Committee for Genetic Studies of Xi' an Jiaotong University.

\section{Genotyping}

Blood was drawn from peripheral veins with vacutainer tubes containing ethylenediaminetetraacetic acid (EDTA). Genomic DNA was extracted from blood leukocytes by the Blood Genomic DNAExtraction System kit(TIANGEN Biotech, Foster City, Beijing, China). The sequences of the rs362719 were retrieved from the National Center for Biotechnology Information (NCBI) database and the primers for rs362719 polymorphisms were designed using the Primer 5.0 program (http://www.premierbiosoft.com/primerdesign/index.html). The primers for rs362719 analysis were: 5'-GGTGCTTATTTTACAGGTTGGA-3' and 5'-CTGGAGATTGAGGAATGGAGTT-3'. Genotyping was performed for rs362719 by polymerase chain reaction (PCR)-restriction fragment length polymorphism (RFLP) methods. PCR was performed using a DNA thermal cycler (Bio-Rad, Hercules, CA, USA). The PCR amplification system had a total volume of $12 \mu \mathrm{L}$, containing 50 200 ng genome DNA, 1X PCR buffer, $15 \mathrm{mM} \mathrm{MgCl}_{2}, 5 \mu \mathrm{M}$ primer, $0.5 \mathrm{U}$ Tag Enzyme (TIANGEN). The PCR products were digested using the HindIII restriction enzyme (New English Biolabs, Beijing, China) at $37^{\circ} \mathrm{C}$ overnight. The PCR product (353 bp) with the A allele could be digested into two fragments ( 272 and $81 \mathrm{bp}$ ), while the PCR product with the C allele could not be separated by HindIII. The digested DNA fragments were separated by electrophoresis on $2.5 \%$ agarose gel in a $0.5 \mathrm{X}$ TBE running buffer. After electrophoresis, the bands were visualized by ethidium bromide staining. A gel imaging system (Quantity one; Bio-Rad) was used to capture the images and analyze the results. For quality control, all genotypes were blinded to the researcher in the genotyping process. Five percent of the samples were repeated for the genotyping assay and the corresponding results were $100 \%$ concordant.

\section{Statistical analysis}

The online SHEsis software (http://analysis.bio-x.cn) (Shi and He, 2005) was used to test for Hardy-Weinberg equilibrium, allele and genotype frequencies. The odds ratios (OR) and $95 \%$ confidence intervals (CI) were estimated for the effects of high risk genotypes and alleles. Differences in gender, allele frequency, and genotype distribution were evaluated using the chisquare test. Demographic and clinical characteristics were analyzed using the chi-square test. All tests were two-tailed and a P value less than 0.05 was set as the significance level. 


\section{RESULTS}

The allele and genotype frequencies of rs362719 in the schizophrenia patients and controls are summarized in Table 1. The allelic distribution frequencies and genotype among both patients and controls were within the Hardy-Weinberg equilibrium. A significant difference was found in the allelic frequencies of rs362719 between schizophrenia patients and healthy controls $\left(\chi^{2}=4.725\right.$, d.f. $\left.=1, \mathrm{P}=0.0297, \mathrm{OR}=0.7842,95 \% \mathrm{CI}=0.6297 \sim 0.9766\right)$. The A allele was more frequent in schizophrenia patients than in healthy controls. As a GWAS on schizophrenia reported robust association between RELN (SNP rs7341475) and schizophrenia in females alone (Shifman et al., 2008), we performed another $\chi^{2}$ analysis of our SNP stratified by gender. The SNP rs362719 was found to have a significant difference in females at the allele level $\left(\chi^{2}=5.748\right.$, d.f. $=1, \mathrm{P}=0.0165$, OR $\left.=0.6860,95 \% \mathrm{CI}=0.5038 \sim 0.9342\right)$, and this association was not observed in males $(\mathrm{P}>0.05)$. To assess the possibility of genotype differences in background variables, the genotypes of rs362719 in schizophrenia patients were classified into three sub-groups: CC genotype, CA genotype and AA genotype. In addition, we also compared the characteristics including age, age at onset, education, number of episodes, patients with a first episode of schizophrenia, as well as patients by each genotype (see Table 2). No significance level was found in the clinical characteristics of schizophrenia patients among the three genotypes (see Table 2).

Table 1. Genotypes and allele frequencies of the rs362719 of the RELN gene in schizophrenia cases and controls.

\begin{tabular}{|c|c|c|c|c|c|c|c|c|c|}
\hline \multirow[t]{2}{*}{ Groups (N) } & \multicolumn{3}{|c|}{ Genotype (N, \%) } & \multirow[t]{2}{*}{$\chi^{2}$} & \multirow[t]{2}{*}{$\mathrm{P}$} & \multicolumn{2}{|c|}{ Allele (N, \%) } & \multirow[t]{2}{*}{$\chi^{2}$} & \multirow[t]{2}{*}{$\mathrm{P}$} \\
\hline & $\mathrm{CC}$ & $\mathrm{CA}$ & $\mathrm{AA}$ & & & $\mathrm{C}$ & $\mathrm{A}$ & & \\
\hline Patients (405) & $191(47.3)$ & $180(44.3)$ & $34(8.4)$ & 4.958 & 0.0838 & $564(69.5)$ & $248(30.5)$ & 4.725 & $0.0297 *$ \\
\hline Control subjects (390) & $214(54.9)$ & $152(39.0)$ & $24(6.2)$ & & & $580(74.4)$ & $200(25.6)$ & & \\
\hline Male-cases (205) & $100(48.8)$ & $94(45.9)$ & $11(5.4)$ & 0.755 & 0.6855 & $294(71.7)$ & $116(28.3)$ & 0.515 & 0.4730 \\
\hline Male-controls (194) & $103(53.1)$ & $81(41.8)$ & $10(5.2)$ & & & $287(74.0)$ & $101(26.0)$ & & \\
\hline Female-cases (200) & $91(45.5)$ & $86(43.0)$ & $23(11.5)$ & 5.563 & 0.0620 & $268(67)$ & $132(33)$ & 5.748 & $0.0165 * *$ \\
\hline Female-controls (196) & $111(56.6)$ & $71(36.2)$ & $14(7.1)$ & & & $293(74.7)$ & $99(25.3)$ & & \\
\hline
\end{tabular}

*Significance level for differences in allele frequency between cases and controls $(\mathrm{P}<0.05)$. **Significance level for differences in allele frequency between female cases and controls $(\mathrm{P}<0.05)$ but not between male cases and controls $(\mathrm{P}>0.05)$.

Table 2. Demographic and clinical characteristics of rs362719 genotype groups within the schizophrenia patients.

\begin{tabular}{lccc}
\hline Characteristics & $\mathrm{CC}(\mathrm{N}=191)$ & $\mathrm{CA}(\mathrm{N}=180)$ & $\mathrm{AA}(\mathrm{N}=34)$ \\
\hline Age (years) & $42.0 \pm 6.7$ & $41.7 \pm 7.9$ & $41.4 \pm 5.6$ \\
Age at onset & $24.6 \pm 9.8$ & $23.4 \pm 8.7$ & $22.5 \pm 8.2$ \\
Years of education & $11.8 \pm 7.4$ & $11.0 \pm 6.1$ & $10.2 \pm 6.9$ \\
Patients with a first episode (in \%) & $57 \%$ & $54 \%$ & $51 \%$ \\
Number of episodes & $1.8 \pm 1.1$ & $1.9 \pm 1.2$ & $2.0 \pm 1.3$ \\
\hline
\end{tabular}

Data are reported as means $\pm \mathrm{SD}$, unless otherwise explained. No significance level was found in demographic and clinical characteristics of schizophrenia patients among the three genotypes.

\section{DISCUSSION}

Given an overlap between bipolar disorder and schizophrenia in clinical symptoms, we postulated that a gene variant involved in bipolar disorder susceptibility may also be ex- 
pressed in patients with schizophrenia. Therefore, we investigated the re362719 variant in the Chinese Han population. We found a positive association between the presence of a $\mathrm{C}$ to $\mathrm{A}$ substitution in rs362719 and a risk of schizophrenia. The allele was over represented among patients with schizophrenia (allele frequencies: 0.305 in cases $v s 0.256$ in controls) in the present study, and demographic and clinical characteristics of schizophrenia patients showed no significant difference among the different genotypes. Therefore, this study may provide direct evidence for the association between the RELN SNP rs362719 and schizophrenia in the Chinese Han population. The association observed in this study was unlikely to be due to population stratification, since we collected data in a relatively narrow region in the Shaanxi province in China, and our samples had adequate power to detect major differences in genetic polymorphisms. Based on our results, rs362719 of the RELN gene may be one of the risk loci in the development of schizophrenia.

Polymorphism of rs362719 has been investigated in several association studies of some other diseases such as autism and childhood epilepsy in an Indian population; however, no association was detected in these studies. In a recent report, rs362719 was found to be associated with a susceptibility to bipolar disorder in women but still may not be replicated (Goes et al., 2010). Since schizophrenia and bipolar disorder are closely related psychiatric disorders, it is encouraging to see a similar effect of rs362719 as regards the predisposition to schizophrenia. To our knowledge, most of the GWAS on schizophrenia and bipolar disorder used the Affymetrix platform, which does not have an assay available for the rs362719 SNP. Only one published research has studied this SNP but no association was found (Need et al., 2009). However, as mentioned by those authors, the sample size was not large enough to identify risk factors of small effects in a genome-wide context (Need et al., 2009).

Reelin-signaling pathway has been found to participate in neuronal migration processes during early brain development and RELN may act as a potential candidate gene for schizophrenia. The Reelin protein is composed of a distinctive N-terminal domain followed by eight tandem repeats comprising an extracellular growth factor (EGF)-like domain, which is surrounded by two sub-repeats. Rs362719 is located at 86 bp upstream from exon 42 and contributes to the EGFlike domain of the fifth reelin repeat (Royaux et al., 1997). In some in vitro studies, it has been reported that three to six (R3-6) repeat fragments of the EGF-like domain can bind to ApoER2 and VLDLR, which are involved in neuron signal transduction (Jossin et al., 2004; Chameau et al., 2009). Moreover, addition of the repeat 5-6 fragments is sufficient to induce phosphorylation of Dab1 (Yasui et al., 2007), which is an "adapter molecule" that transduces the signal to the next molecule in the cytosol and is responsible for triggering of the reelin intracellular signaling pathway. These studies indicate that this risk allele maybe within a regulatory element.

Interestingly, rs362719 allele distributions and genotype were analyzed separately for men and women. As showed in Table 1, the A allele frequency of rs362719 in schizophrenia female population was significantly higher in patients with schizophrenia $(33 \%)$ than that in controls (25.3\%); however, the A allele frequency of rs362719 was similar in male schizophrenia populations $(28.3 \%)$ and controls $(26.0 \%)$. These results may suggest a gender-specific mechanism in the development of schizophrenia. While gender difference in schizophrenia has been found in previous studies, so far it is still unclear as to the underlying molecular mechanisms. A previous meta-analysis also presented evidence for gender difference in schizophrenia risk (Aleman et al., 2003). A GWAS on schizophrenia (Shifman et al., 2008) also reported that a common DNA variant (rs7341475) was associated with an increased risk of schizophrenia in 
Ashkenazi Jewish females but not males. The effect of gender has also been reported in another study, which showed that RELN expression was higher in women compared to men (in layer I neurons), and a reduction of RELN expression was observed in men with schizophrenia alone (in the superficial interstitial white matter neurons) (Eastwood and Harrison, 2003). However, as mentioned by these authors, despite the small sample involved this has not been observed in other brain regions. Rat studies have shown that progesterone, a female hormone, robustly up-regulated the expression of Reelin in peripheral nerves (Roglio et al., 2008). In another animal study, the heterozygous reeler mouse was also found to have diminished expression of the oxytocin receptor (Liu et al., 2005). Since oxytocin receptor is key regulator of the most well-characterized sexually dimorphic signaling pathways in the brain (de Vries, 2008), this may serve as a molecular basis for gender differences observed in humans. In addition, it was observed that the only one functional RELN gene resulted in loss of Purkinje cells in male mice but not in reelin-deficient female mice (Hadj-Sahraoui et al., 1996). These results of femalespecific association of RELN with schizophrenia suggest a possible pathway where sex hormones modulate gene expression in brain, thereby increasing the susceptibility to psychosis.

In summary, the results of the current investigation showed an association between polymorphisms rs362719 in the RELN gene and risk of schizophrenia in the Chinese Han female population. Moreover, our study also suggested a possible gender-specific mechanism of schizophrenia development. Further investigations of genetic susceptibility among different ethnic populations are also required to elucidate the role of the RELN polymorphisms on the increase risk of schizophrenia in the future.

\section{ACKNOWLEDGMENTS}

The author would like to thank all patients and healthy volunteers for their participation in this research and all medical staff members involved in diagnosis and sample-collecting. Research supported by fund of Genetic Resource of Chinese Population (\#57004). We also specially thank Bo Xing for his useful comments during the preparation of this manuscript.

\section{REFERENCES}

Abdolmaleky HM, Cheng KH, Russo A, Smith CL, et al. (2005). Hypermethylation of the reelin (RELN) promoter in the brain of schizophrenic patients: a preliminary report. Am. J. Med. Genet. B Neuropsychiatr. Genet. 134B: 60-66.

Aleman A, Kahn RS and Selten JP (2003). Sex differences in the risk of schizophrenia: evidence from meta-analysis. Arch. Gen. Psychiatry 60: 565-571.

American Psychiatric Association (2000). Diagnostic and Statistical Manual of Mental Disorders. American Psychiatric Association, Washington.

Badner JA and Gershon ES (2002a). Meta-analysis of whole-genome linkage scans of bipolar disorder and schizophrenia. Mol. Psychiatry 7: 405-411.

Badner JA and Gershon ES (2002b). Regional meta-analysis of published data supports linkage of autism with markers on chromosome 7. Mol. Psychiatry 7: 56-66.

Berrettini W (2003). Evidence for shared susceptibility in bipolar disorder and schizophrenia. Am. J. Med. Genet. C Semin. Med. Genet. 123C: 59-64.

Cardno AG and Gottesman II (2000). Twin studies of schizophrenia: from bow-and-arrow concordances to star wars Mx and functional genomics. Am. J. Med. Genet. 97: 12-17.

Cardno AG, Rijsdijk FV, Sham PC, Murray RM, et al. (2002). A twin study of genetic relationships between psychotic symptoms. Am. J. Psychiatry 159: 539-545.

Chameau P, Inta D, Vitalis T, Monyer H, et al. (2009). The N-terminal region of reelin regulates postnatal dendritic 
maturation of cortical pyramidal neurons. Proc. Natl. Acad. Sci. U. S. A. 106: 7227-7232.

Chen Y, Beffert U, Ertunc M, Tang TS, et al. (2005). Reelin modulates NMDA receptor activity in cortical neurons. $J$. Neurosci. 25: 8209-8216.

Clinton SM and Meador-Woodruff JH (2004). Abnormalities of the NMDA receptor and associated intracellular molecules in the thalamus in schizophrenia and bipolar disorder. Neuropsychopharmacology 29: 1353-1362.

Craddock N and Owen MJ (2005). The beginning of the end for the Kraepelinian dichotomy. Br. J. Psychiatry 186: 364-366. de Vries GJ (2008). Sex differences in vasopressin and oxytocin innervation of the brain. Prog. Brain Res. 170: 17-27.

Dong E, Guidotti A, Grayson DR and Costa E (2007). Histone hyperacetylation induces demethylation of reelin and 67kDa glutamic acid decarboxylase promoters. Proc. Natl. Acad. Sci. U. S. A. 104: 4676-4681.

Dong E, Nelson M, Grayson DR, Costa E, et al. (2008). Clozapine and sulpiride but not haloperidol or olanzapine activate brain DNA demethylation. Proc. Natl. Acad. Sci U. S. A. 105: 13614-13619.

Eastwood S and Harrison P (2003). Interstitial white matter neurons express less reelin and are abnormally distributed in schizophrenia: towards an integration of molecular and morphologic aspects of the neurodevelopmental hypothesis. Mol. Psychiatry 8: 821-831.

Fatemi SH, Earle JA and McMenomy T (2000). Reduction in Reelin immunoreactivity in hippocampus of subjects with schizophrenia, bipolar disorder and major depression. Mol. Psychiatry 5: 654-663.

Goes FS, Willour VL, Zandi PP, Belmonte PL, et al. (2010). Sex-specific association of the Reelin gene with bipolar disorder. Am. J. Med. Genet. B Neuropsychiatr. Genet. 153B: 549-553.

Grayson DR, Jia X, Chen Y, Sharma RP, et al. (2005). Reelin promoter hypermethylation in schizophrenia. Proc. Natl. Acad. Sci. U. S. A. 102: 9341-9346.

Guidotti A, Auta J, Davis JM, Di-Giorgi-Gerevini V, et al. (2000). Decrease in reelin and glutamic acid decarboxylase67 (GAD67) expression in schizophrenia and bipolar disorder: a postmortem brain study. Arch. Gen. Psychiatry 57: 1061-1069.

Hadj-Sahraoui N, Frederic F, Delhaye-Bouchaud N and Mariani J (1996). Gender effect on Purkinje cell loss in the cerebellum of the heterozygous reeler mouse. J. Neurogenet. 11: 45-58.

Herz J and Chen Y (2006). Reelin, lipoprotein receptors and synaptic plasticity. Nat. Rev. Neurosci. 7: 850-859.

Jossin Y, Ignatova N, Hiesberger T, Herz J, et al. (2004). The central fragment of Reelin, generated by proteolytic processing in vivo, is critical to its function during cortical plate development. J. Neurosci. 24: 514-521.

Lander E and Kruglyak L (1995). Genetic dissection of complex traits: guidelines for interpreting and reporting linkage results. Nat. Genet. 11: 241-247.

Lichtenstein P, Yip BH, Bjork C, Pawitan Y, et al. (2009). Common genetic determinants of schizophrenia and bipolar disorder in Swedish families: a population-based study. Lancet 373: 234-239.

Liu W, Pappas GD and Carter CS (2005). Oxytocin receptors in brain cortical regions are reduced in haploinsufficient (+/-) reeler mice. Neurol. Res. 27: 339-345.

McGuffin P, Rijsdijk F, Andrew M, Sham P, et al. (2003). The heritability of bipolar affective disorder and the genetic relationship to unipolar depression. Arch. Gen. Psychiatry 60: 497-502.

Mill J, Tang T, Kaminsky Z, Khare T, et al. (2008). Epigenomic profiling reveals DNA-methylation changes associated with major psychosis. Am. J. Hum. Genet. 82: 696-711.

Moller HJ (2003). Bipolar disorder and schizophrenia: distinct illnesses or a continuum? J. Clin. Psychiatry 64 (Suppl 6): 23-27.

Moskvina V, Craddock N, Holmans P, Nikolov I, et al. (2009).Gene-wide analyses of genome-wide association data sets: evidence for multiple common risk alleles for schizophrenia and bipolar disorder and for overlap in genetic risk. Mol. Psychiatry 14:252-260.

Murray RM, Sham P, van Os J, Zanelli J, et al. (2004). A developmental model for similarities and dissimilarities between schizophrenia and bipolar disorder. Schizophr. Res. 71: 405-416.

Need AC, Ge D, Weale ME, Maia J, et al. (2009). A genome-wide investigation of SNPs and CNVs in schizophrenia. PLoS Genet. 5: e1000373.

Quattrocchi CC, Wannenes F, Persico AM, Ciafre SA, et al. (2002). Reelin is a serine protease of the extracellular matrix. J. Biol. Chem. 277: 303-309.

Roglio I, Bianchi R, Gotti S, Scurati S, et al. (2008). Neuroprotective effects of dihydroprogesterone and progesterone in an experimental model of nerve crush injury. Neuroscience 155: 673-685.

Royaux I, Lambert de RC, D’Arcangelo G, Demirov D, et al. (1997). Genomic organization of the mouse reelin gene. Genomics 46: 240-250.

Shi YY and He L (2005). SHEsis, a powerful software platform for analyses of linkage disequilibrium, haplotype construction, and genetic association at polymorphism loci. Cell Res. 15: 97-98.

Shifman S, Johannesson M, Bronstein M, Chen SX, et al. (2008). Genome-wide association identifies a common variant in the reelin gene that increases the risk of schizophrenia only in women. PLoS Genet. 4: e28. 
Tochigi M, Iwamoto K, Bundo M, Komori A, et al. (2008). Methylation status of the reelin promoter region in the brain of schizophrenic patients. Biol. Psychiatry 63: 530-533.

Tsuang MT, Taylor L and Faraone SV (2004). An overview of the genetics of psychotic mood disorders. J. Psychiatr. Res. 38: 3-15.

van Os J, Gilvarry C, Bale R, van Horn E, et al. (1999). A comparison of the utility of dimensional and categorical representations of psychosis. UK700 Group. Psychol. Med. 29: 595-606.

Veldic M, Kadriu B, Maloku E, Agis-Balboa RC, et al. (2007). Epigenetic mechanisms expressed in basal ganglia GABAergic neurons differentiate schizophrenia from bipolar disorder. Schizophr. Res. 91: 51-61.

Wedenoja J, Loukola A, Tuulio-Henriksson A, Paunio T, et al. (2008). Replication of linkage on chromosome 7q22 and association of the regional Reelin gene with working memory in schizophrenia families. Mol. Psychiatry 13: 673-684.

Yasui N, Nogi T, Kitao T, Nakano Y, et al. (2007). Structure of a receptor-binding fragment of reelin and mutational analysis reveal a recognition mechanism similar to endocytic receptors. Proc. Natl. Acad. Sci. U. S. A. 104: 9988-9993. 